\title{
Vers une prise en charge intégrée des sarcomes
}

\section{Progress towards Integrated Treatment of Sarcomas}

\author{
B. Bui-Nguyen \\ (C) Lavoisier SAS 2016
}

Ce numéro d'Oncologie aborde quelques aspects des sarcomes, un domaine dans le cours d'un bouleversement commencé il y a une vingtaine d'années, avec le début du décryptage génétique de ces tumeurs dans des laboratoires comme celui d'Alain Aurias à l'institut Curie et avec la formalisation de groupes cliniques nationaux et internationaux dédiés, comme le GSF-GETO en France. Ce sont, on le sait, des maladies rares : on peut estimer à 3500 par an le nombre de nouveaux cas de sarcomes en France ; 75 \% sont des sarcomes des tissus mous de toutes localisations, $15 \%$ sont des sarcomes viscéraux, $10 \%$ des sarcomes osseux et, si la médiane d'âge se situe entre 60 et 65 ans, $3 \%$ concernent des enfants et adolescents ; la spécificité de ces tumeurs pédiatriques mérite qu'elles soient envisagées à part. La diversité clinique des sarcomes s'accompagne d'une grande diversité histologique. Rareté et hétérogénéité expliquent les difficultés rencontrées à toutes les étapes de la prise en charge.

Le premier problème est diagnostique, et il est simplement de penser qu'une masse puisse être un sarcome. Les lésions tumorales ou pseudotumorales des parties molles sont en effet très majoritairement bénignes, dans un rapport malin/bénin de $1 / 200$ à 1/300. En conséquence, un tiers seulement des sarcomes sont suspectés avant une tentative d'exérèse et vont bénéficier d'une prise en charge préchirurgicale adéquate, avec imagerie, biopsie et planification thérapeutique d'emblée dans une concertation pluridisciplinaire dédiée [1]. Il faut donc impérativement garder à l'esprit la règle simple qu'une masse des tissus mous de plus de $5 \mathrm{~cm}$, a fortiori profonde et évolutive, peut être un sarcome (le risque monte alors à 1/7). L'enjeu est majeur pour le patient, comme souligné dans l'article de S. Bonvalot, car de la qualité de la première prise en charge, en premier lieu de la qualité de la chirurgie, dépendent pour une large part le pronostic vital et le résultat fonctionnel.

Le diagnostic anatomopathologique repose en pratique sur la morphologie et l'immunohistochimie. Les tumeurs mésen-

B. Bui-Nguyen $(\bowtie)$

Institut Bergonié, 229, cours de l'Argonne,

F-33076 Bordeaux, France

e-mail : B.Bui@bordeaux.unicancer.fr chymateuses sont un domaine difficile, en attestent les 9$10 \%$ de discordances majeures (malin/bénin, sarcome/nonsarcomes) retrouvées dans les relectures histologiques systématiques, sans parler des discordances de classifications. La biologie moléculaire a contribué de manière cruciale et déterminante à la classification actuelle des sarcomes et continue à le faire, ainsi que le montre S. le Guellec. Elle est surtout devenue désormais indispensable dans le plateau technique anatomopathologique des sarcomes, comme outil diagnostique, voire pronostique. De ce point de vue, si le grade histopronostique reste le facteur pronostique prédominant, il est très probable que la signature CINSARC, adaptée pour être déterminable sur tissus en paraffine, la supplantera dans les années qui viennent; son rôle prédictif dans la prévision de l'efficacité des traitements sera aussi à déterminer.

La prise en charge thérapeutique des sarcomes pose encore de nombreuses questions générales et plus encore de questions spécifiques si l'on considère les types, les localisations ou les terrains. Les rhabdomyosarcomes embryonnaires de l'enfant, les ostéosarcomes et les sarcomes d'Ewing ont bénéficié depuis plus de 40 ans de l'apport de chimiothérapies (néo)adjuvantes. Mais les résultats plafonnent depuis avec la persistance de 25 à $30 \%$ d'échecs thérapeutiques. Pour les sarcomes des tissus mous de l'adulte, la survie globale est actuellement de 60-65\%. Il faut donc progresser. Le premier gisement d'amélioration consiste à optimiser la prise en charge de chaque patient avec les moyens dont nous disposons, notamment pour les patients sans métastase initiale, qui représentent $85-90 \%$ des cas. L'organisation de réseaux pour les cancers de l'enfant et pour les tumeurs rares a permis la mise à disposition de relectures anatomopathologiques systématiques qui ont montré leur utilité, nous l'avons vu, et aussi leur efficience en termes médicoéconomiques [2]. Les réseaux SFCE, RESOS et NETSARC offrent aussi des concertations pluridisciplinaires dédiées, régionales et interrégionales et mettent à disposition les compétences spécifiques et les plateaux techniques nécessaires. Ces efforts d'organisation prouvent leur utilité : une amélioration significative de la survie globale a été retrouvée avec le recours aux concertations pluridisciplinaires [3] et plus largement, la prise en charge au sein de NETSARC, pour les patients atteints d'un 
sarcome des tissus mous [4] ; ce réseau fait l'objet d'une présentation ici par Ducimetiere et al. Pour ces tumeurs, le progrès en survie obtenu est équivalent à celui espéré mais non atteint dans des essais de chimiothérapie adjuvante comme celui de l'EORTC [5].

Dans les sarcomes des tissus mous de l'adulte, l'apport des traitements généraux adjuvants sur le risque évolutif principal constitué par l'évolution métastatique reste controversé et en tout cas limité à des sous-groupes tels que, peutêtre, les sarcomes de haut grade, les femmes, les patients avec une exérèse tumorale R1. Cela reflète l'efficacité limitée des traitements cytotoxiques conventionnels, comme le montre aussi l'évolution des malades métastatiques, qui reste dans l'ensemble médiocre avec une médiane de survie autour de 18 mois. Pour ces patients, aucun traitement n'a à ce jour produit une supériorité en termes de survie globale sur la doxorubicine seule. Les associations, qui donnent des taux de réponse supérieurs, n'ont ainsi toujours d'utilité qu'en éventuelle induction, pour préparer un traitement local, en général une chirurgie. Les méthodes classiques de recherche clinique ont certes abouti récemment à des résultats importants, comme la mise en évidence de l'efficacité de l'antiangiogène pazopanib [6], ce qui a permis d'ailleurs sa mise sur le marché pour le traitement des sarcomes avancés, la seule depuis longtemps. On peut aussi citer l'essai de phase 3 qui vient de montrer l'efficacité de l'érébuline, un agent agissant sur les microtubules et le remodelage vasculaire tumoral, pour les léiomyosarcomes et les liposarcomes [7]. Mais on peut penser que les progrès viendront maintenant d'une intégration encore plus étroite de la recherche clinique avec une recherche plus fondamentale caractérisant les mécanismes pathogéniques tumoraux. Plusieurs illustrations de ce propos peuvent être évoquées dans le domaine des sarcomes, comme le discute ici A. Italiano, mais l'exemple le plus marquant dans le domaine des sarcomes reste le traitement des tumeurs stromales gastrointestinales par imatinib, avec la publication du premier cas traité par Joensuu et al. en 2001, seulement trois ans après la démonstration du rôle pathogénique de c-kit dans ces tumeurs par Hirota et al. Depuis, l'efficacité de traitements ciblés, spécifiques, a pu être montrée dans d'autres types de sarcomes, du dermatofibrosarcome de Darier-Ferrand aux sarcomes myofibroblastiques inflammatoires, en passant par des tumeurs bénignes mais agressives comme les tumeurs à cellules géantes de l'os ou les synovites villonodulaires. D'autres stratégies sont en cours d'évaluation, diri- gées vers d'autres anomalies génétiques spécifiques dans le contrôle du cycle cellulaire (inhibitions de MDM2 et de CDK4, amplifiées dans les liposarcomes différenciés/dédifférenciés) ou la régulation épigénétique (inhibition de EZH2, dérégulé par mutation inactivatrice de INI1 dans les sarcomes rhabdoïdes), ou par des voies différentes telle la régulation de l'immunité antitumorale. Il faut dire, à ce propos, qu'en France la communauté des chercheurs et cliniciens dédiés aux sarcomes du GSF-GETO a, en regard de ces enjeux, mis en œuvre des programmes importants, fondamentaux comme ICGC-léiomyosarcomes (collaboration avec le Consortium international pour la caractérisation génomique des tumeurs), de transfert biologique comme la recherche de nouvelles translocations récurrentes, ou clinique comme MULTISARC (traitement de maladies métastatiques ciblé sur des anomalies détectées par une analyse génomique tumorale systématique).

\section{Références}

1. Mathoulin-Pélissier S, Chevreau C, Bellera C, et al. (2014) Adherence to consensus-based diagnosis and treatment guidelines in adult soft-tissue sarcoma patients: a French prospective population-based study. Ann Oncol 25: 225-31

2. Rossi CR, Vecchiato A, Mastrangelo G, et al. (2013) Adherence to treatment guidelines for primary sarcomas affects patient survival: a side study of the European CONnective TIssue CAncer NETwork (CONTICANET). Ann Oncol 24: 1685-91

3. Perrier L, Kembou Nzale S, Rascle P, et al. (2014) Economic impact of centralized histological reviews in patients with sarcoma, GIST, and desmoid tumors. 17th Annual European Congress of the International Society for Pharmacoeconomics and Outcomes Research (ISPOR). Poster presentation (Value in Health, vol 17, number 7, page A624). RAI, Amsterdam, The Netherlands

4. Ducimetiere F, Le Cesne A, Bui-Nguyen B, et al. (2013) NETSARC: a clinical reference sarcoma centers network of the French Sarcoma Group. Connective Tissue Oncology Society (CTOS) Annual meeting, New York

5. Woll PJ, Reichardt P, Le Cesne A, et al. (2012) Adjuvant chemotherapy with doxorubicin, ifosfamide, and lenograstim for resected soft-tissue sarcoma (EORTC 62931): a multicentre randomised controlled trial. Lancet Oncol 13: 1045-54

6. van der Graaf WT, Blay JY, Chawla SP, et al. (2012) Pazopanib for metastatic soft-tissue sarcoma (PALETTE): a randomised, doubleblind, placebo-controlled phase 3 trial. Lancet 379: 1879-86

7. Schöffski P, Chawla S, Maki RG, et al. (2016) Eribulin versus dacarbazine in previously treated patients with advanced liposarcoma or leiomyosarcoma: a randomised, open-label, multicentre, phase 3 trial. Lancet 387: 1629-37 